\title{
A three-gene predictor for early intrahepatic recurrence of hepatocellular carcinoma after curative hepatectomy
}

\author{
HIDEAKI SOMURA $^{1 *}$, NORIO IIZUKA ${ }^{1,2^{*}}$, TAKAO TAMESA ${ }^{1}$, KAZUHIKO SAKAMOTO ${ }^{1}$, \\ TAKASHI HAMAGUCHI ${ }^{1}$, RYOUICHI TSUNEDOMI ${ }^{1}$, HISAFUMI YAMADA-OKABE ${ }^{3}$, MIKIKO SAWAMURA $^{4}$, \\ MASAYA ERAMOTO ${ }^{4}$, TAKANOBU MIYAMOTO ${ }^{4}$, YOSHIHIKO HAMAMOTO $^{4}$ and MASAAKI OKA $^{1}$ \\ Departments of ${ }^{1}$ Surgery II, and ${ }^{2}$ Complementary Medicine of Applied Molecular Bioscience, Yamaguchi University \\ Graduate School of Medicine, 1-1-1 Minami-Kogushi, Ube, Yamaguchi 755-8505; ${ }^{3}$ Pharmaceutical Research \\ Department 4, Kamakura Research Laboratories, Chugai Pharmaceutical Co. Ltd., 200 Kajiwara, Kamakura, \\ Kanagawa 247-8530; ${ }^{4}$ Department of Biomolecular Engineering of Applied Molecular Bioscience, \\ Yamaguchi University Graduate School of Medicine, 2-16-1 Tokiwadai, Ube, Yamaguchi 755-8611, Japan
}

Received July 30, 2007; Accepted September 24, 2007

\begin{abstract}
We previously developed a DNA microarray-based system that out-performs traditionally used clinical parameters for prediction of early intrahepatic recurrence (IHR) of hepatocellular carcinoma (HCC). Because DNA microarray is too expensive for daily clinical use, we used a quantitative realtime reverse transcription-polymerase chain reaction (QRTPCR) to develop a lower-cost predictor for early IHR. From the 12 early IHR-related genes integrated in the previous predictor, we selected 6 genes whose levels showed the strongest association between data from the 2 distinct DNA microarray platforms with the same sample set. Expression of these 6 genes relative to that of $G A P D H$ was measured by QRT-PCR in 82 HCCs. Of the 82 HCCs, 39 and 43 were assigned to training and independent test sets, respectively. By searching all combinations $(n=2-6)$ of the 6 genes, we found an optimal combination of 3 genes (HLADRA, DDX17 and LAPTM5) that minimized the leave-one-out error for prediction of early IHR in the training set. The 3-gene predictor constructed with the Fisher linear classifier correctly predicted early IHR or non-recurrence in $35(81.4 \%)$ of 43 HCCs in the independent test set and had a high positive predictive value of $72.7 \%$ and a high negative predictive value of $84.4 \%$. Multivariate analysis with the stepwise logistic regression showed that the 3 -gene predictor $[\mathrm{F}(\mathrm{x})<0]$ was an independent risk
\end{abstract}

Correspondence to: Dr M. Oka, Department of Surgery II, Yamaguchi University Graduate School of Medicine, 1-1-1 Minami-Kogushi, Ube, Yamaguchi 755-8505, Japan

E-mail:2geka-1@po.cc.yamaguchi-u.ac.jp

${ }^{*}$ Contributed equally

Key words: hepatocellular carcinoma, microarray, RT-PCR, intrahepatic recurrence, surgery factor for early IHR (risk ratio, 13.6; $\mathrm{p}=0.006$ ), indicating its potential as an easy-to-use predictor for accurate prediction of early IHR of HCC.

\section{Introduction}

Hepatocellular carcinoma (HCC) is one of the most common cancers, with an estimated 564,000 new cases worldwide in 2000 (1), and represents a major international health problem because the incidence is increasing in many countries $(2,3)$. Major obstacles in the surgical treatment of HCC are the low resectability rate at time of diagnosis and the high rate of intrahepatic recurrence (IHR) after treatment. Indeed, IHR is observed in $30-50 \%$ of HCC patients even after curative surgery (4-6). There are two representative modes of IHR after surgery, early IHR and late IHR (3-6). Most cases of early IHR can be attributed to intrahepatic metastasis of cancer cells and are detected within 1 to 2 years of surgery. However, early IHR limits the potential for surgical cure of HCC $(6,7)$. To avoid unnecessary overtreatment of patients who have been cured by surgery alone and to provide more personalized therapeutic options, we must accurately predict early IHR or non-recurrence. Accurate prediction of early IHR may lead to an improvement of the prognoses of HCC.

In the post-genomic era, DNA microarray technology has created a new avenue in the research field of biomedical science (8). One can now use DNA microarray technology, which provides a snapshot of comprehensive genetic alterations, not only to gain insights into the molecular basis of cancer biology, but also to stratify cancer patients according to their clinical course. Several researchers, including ourselves, have attempted to predict postoperative recurrence of HCC by means of genome-wide information from DNA microarray analysis $(6,7,9)$. Prior to clinical use of microarrays as multiplegene predictors, the predictive performance must be validated in a larger cohort (10); however, none of the previous studies $(6,7,9)$ addressed this issue. One possible reason is that arraybased strategies are too expensive for confirmation studies or routine clinical use, and therefore such an expensive strategy 


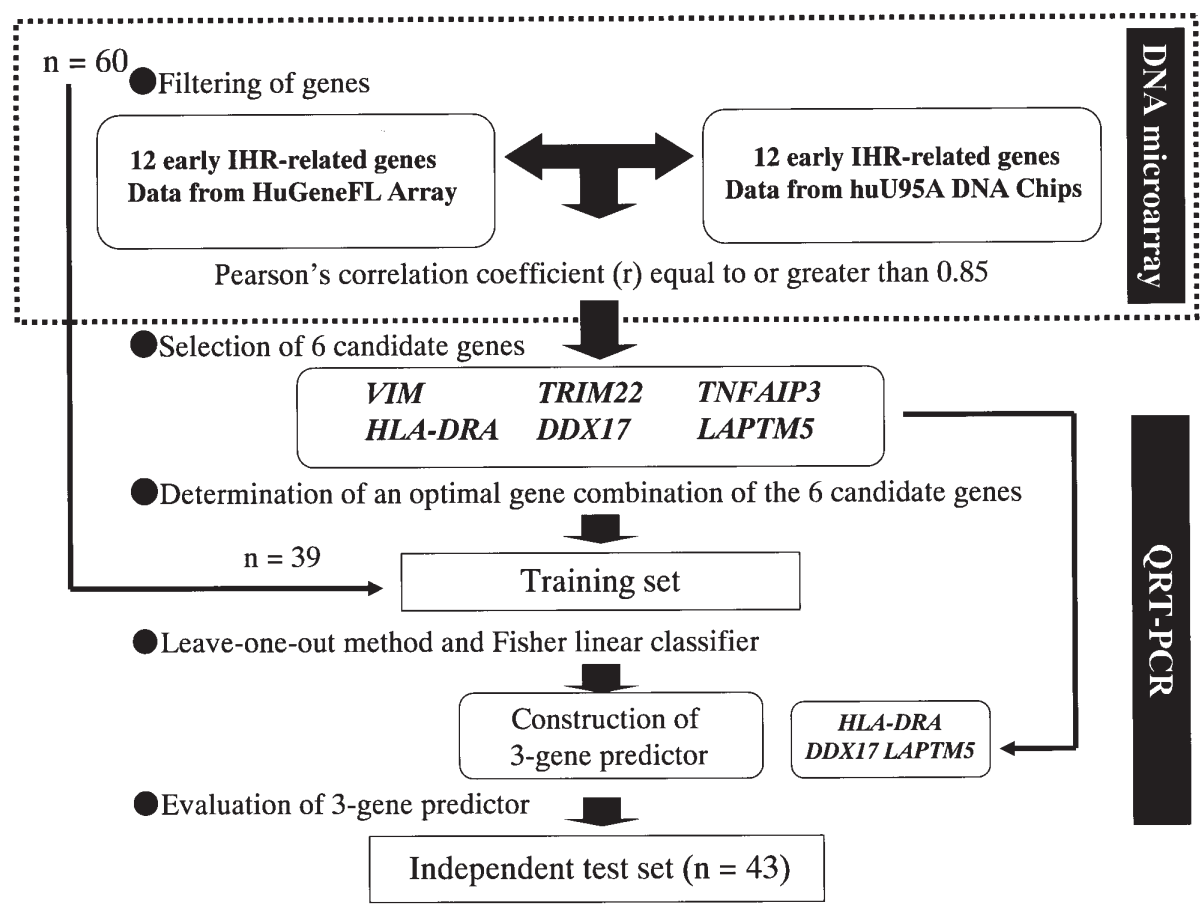

Figure 1. Schematic diagram of the design our study. For HuGeneFL Array, the clinicopathologic features and gene expression data of the $60 \mathrm{HCCs}$ are available on our website (http://surgery2.med.yamaguchi-u.ac.jp/ research/DNAchip/hcc-recurrence/index.html). QRT-PCR, quantitative real-time RT-PCR.

must be replaced with a lower-cost method of analysis such as reverse transcription-polymerase chain reaction (RT-PCR).

To address this issue, it is crucial to decrease the number of genes for prediction. In our previous study, we successfully reduced the dimensionality by 12 without loss of predictive power (6). In the present study, we attempted to decrease the number of genes to a minimum without loss of predictive power. We developed a 3-gene quantitative real-time RT-PCR (QRT-PCR) assay that allows simple, inexpensive and accurate prediction of early IHR of HCC.

\section{Materials and methods}

Selection of 6 candidate genes related to early IHR from pooled DNA microarray data. We previously analyzed the expression of 7070 genes in $60 \mathrm{HCC}$ samples using high-density oligonucleotide arrays (HuGeneFL Array, Affymetrix, Santa Clara, CA) and pooled the data (6,11-13). The clinicopathologic features and gene expression data of the $60 \mathrm{HCCs}$ are available on our website (http://surgery2.med.yamaguchi-u.ac.jp/ research/DNAchip/hcc-recurrence/index.html). Our array analysis yielded a robust predictive panel of 12 genes for early IHR (6). More recently, we used a new DNA microarray platform, huU95A DNA Chips ${ }^{\circledR}$ (Affymetrix) to analyze the expression of 12600 genes in RNAs isolated from the same 60 HCCs (14).

As shown in the schematic diagram of our current study (Fig. 1), we evaluated the reproducibility of the 12 early-IHRrelated genes between the 2 microarray platforms described above. Of the 12 genes, we selected 6 candidate genes (TNFAIP3, HLADRA, TRIM22, DDX17, VIM and LAPTM5) whose levels showed strongest association $(\mathrm{r}=\mathrm{or}>0.85)$ between the 2 DNA microarray platforms (Fig. 2). Levels of all 6 genes were significantly lower in HCC with early IHR than in HCC without early IHR. We focused our investigation on the 6 genes and measured their levels by means of quantitative real-time RT-PCR (QRT-PCR).

Subjects. Eighty-two HCC samples from 82 patients were selected from our collection and subjected to QRT-PCR. HCC was histopathologically diagnosed in all 82 patients who underwent curative hepatectomy. Written informed consent was obtained from all patients before surgery. The study protocol was approved by the Institutional Review Board for Human Use at Yamaguchi University School of Medicine (Japan).

Of the $82 \mathrm{HCCs}, 39$, which were included in our previous DNA microarray study (6), were defined as training samples, and the remaining 43 were defined as test samples. Clinicopathologic features of the training and test sets based on the UICC TNM classification are shown in Table I. Patient follow-up was performed as reported previously (6).

QRT-PCR analysis. RNA extraction and RT reaction were carried out as reported previously $(15,16)$. Following the RT step, real-time PCR (LightCycler System Version 3.39; Roche Diagnostics, Mannheim, Germany) was performed with 10 pmol of each primer set (described below), cDNA equivalent to $10 \mathrm{ng}$ initial RNA and $2 \mathrm{ml}$ LightCycler Fast Start DNA Master SYBR-Green I (Roche Diagnostics).

The following real-time PCR protocol was employed: denaturation for $10 \mathrm{~min}$ at $95^{\circ} \mathrm{C}$ and 40 cycles of 10 -sec denaturation at $95^{\circ} \mathrm{C}, 10$-sec annealing at $60^{\circ} \mathrm{C}$ and 20 -sec elongation at $72^{\circ} \mathrm{C}$. We quantified mRNA levels for the 6 experimental genes and GAPDH with the crossing point (CP), which was calculated with the second derivative maximum 


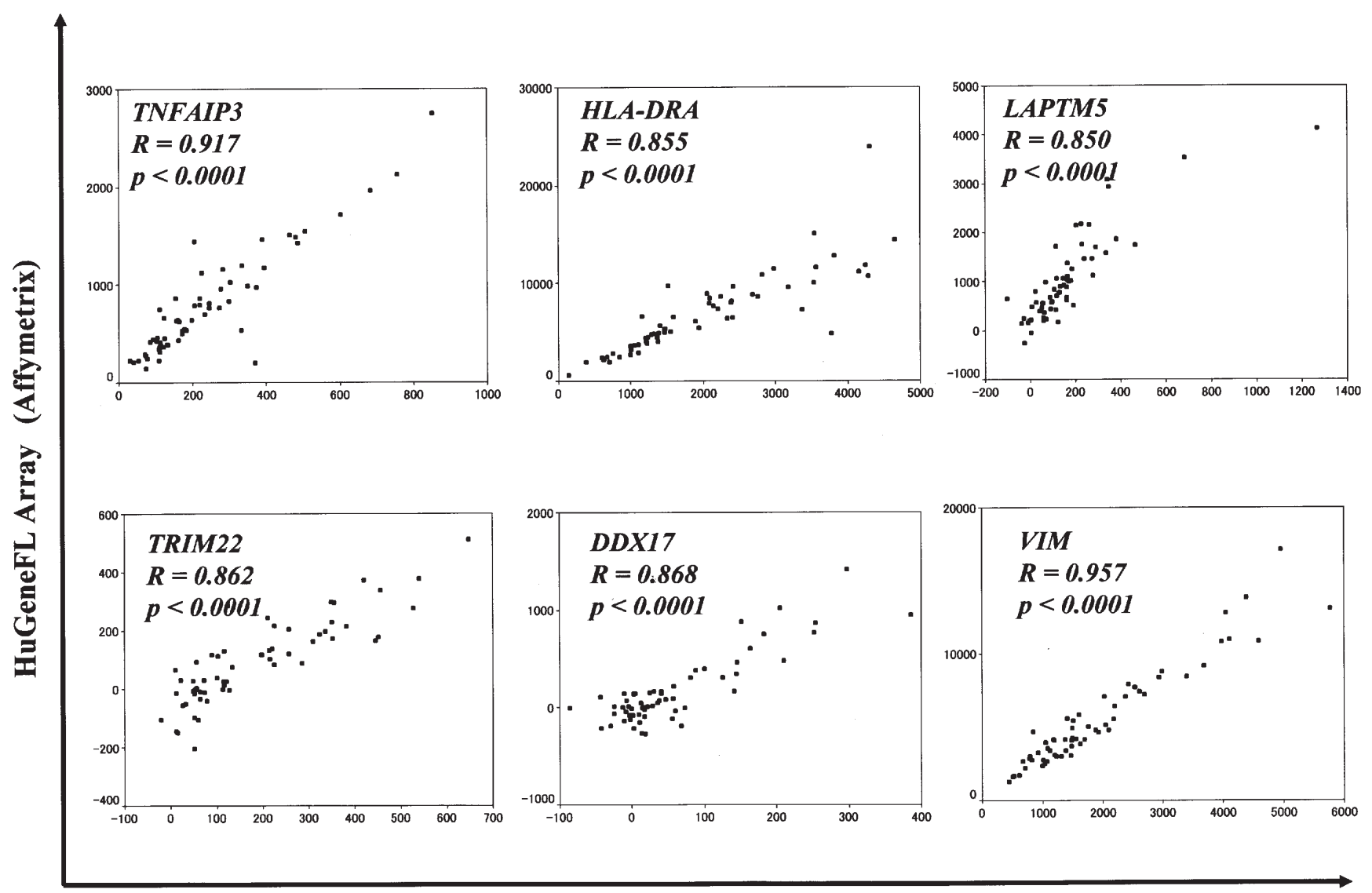

huU95A DNA Chips ${ }^{\circledR}$ (Affymetrix)

Figure 2. Relation of levels of 6 early IHR-related genes between 2 distinct microarray platforms (HuGeneFL Array and huU95A DNA Chips). Gene symbols were obtained from Entrez Gene (http://www.ncbi.nlm.nih.gov/entrez/query.fcgi?db=gene).

Table I. Clinicopathologic features of HCC.

Early intrahepatic recurrence

Training set $(\mathrm{n}=39)$

Test set $(n=43)$

$\overline{\text { Positive }(n=17)}$ Negative $(n=22) \quad$ P-value Positive $(n=13) \quad$ Negative $(n=30) \quad$ P-value

Sex (male/female)

Age in years (mean $\pm \mathrm{SD}$ )

Viral infection (HBV/HCV/non-B non-C)

Tumor size in $\mathrm{cm}($ mean $\pm \mathrm{SD})$

Primary lesion (single/multiple)

Histological grading $(\mathrm{G} 1 / \mathrm{G} 2 / \mathrm{G} 3)^{\mathrm{a}}$

Venous invasion $(-/+)$

Stage (I/II/IIIA) $)^{\text {a }}$

$12 / 5$
$62.4 \pm 9.7$
$4 / 11 / 2$
$5.0 \pm 2.9$
$4 / 13$
$0 / 11 / 6$
$5 / 12$
$2 / 10 / 5$

HBV, hepatitis B virus; HCV, hepatitis C virus; non-B non-C, patient negative for both hepatitis B antigen and HCV antibody. Gl, well differentiated; G2, moderately differentiated; G3, poorly differentiated. Minor, subsegmentectomy and partial hepatectomy. P-value was evaluated by Fisher's exact test, chi-square test or Student's t-test. ${ }^{a}$ Assessment based on pTNM classification of UICC. N.S., not significant. method included in the LightCycler Software 3.39 (Roche Diagnostics), as described previously $(15,16)$. The CP is the number of PCR cycles when maximal acceleration of the increase in fluorescence is reached. We also calculated the PCR efficiency (E) for each gene in titration experiments with various amounts of cDNA according to the method of
Neuvians et al (17). The investigated transcripts showed PCR efficiencies (E) of 1.937 for GAPDH, 2.020 for TNFAIP3, 1.937 for HLADRA, 2.030 for TRIM22, 1.910 for DDX17, 2.010 for VIM and 1.927 for LAPTM5. Target gene expression relative to GAPDH $\left(\mathrm{N}_{\text {target }}\right)$, was determined with the following

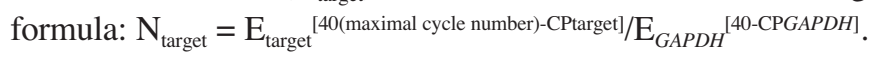



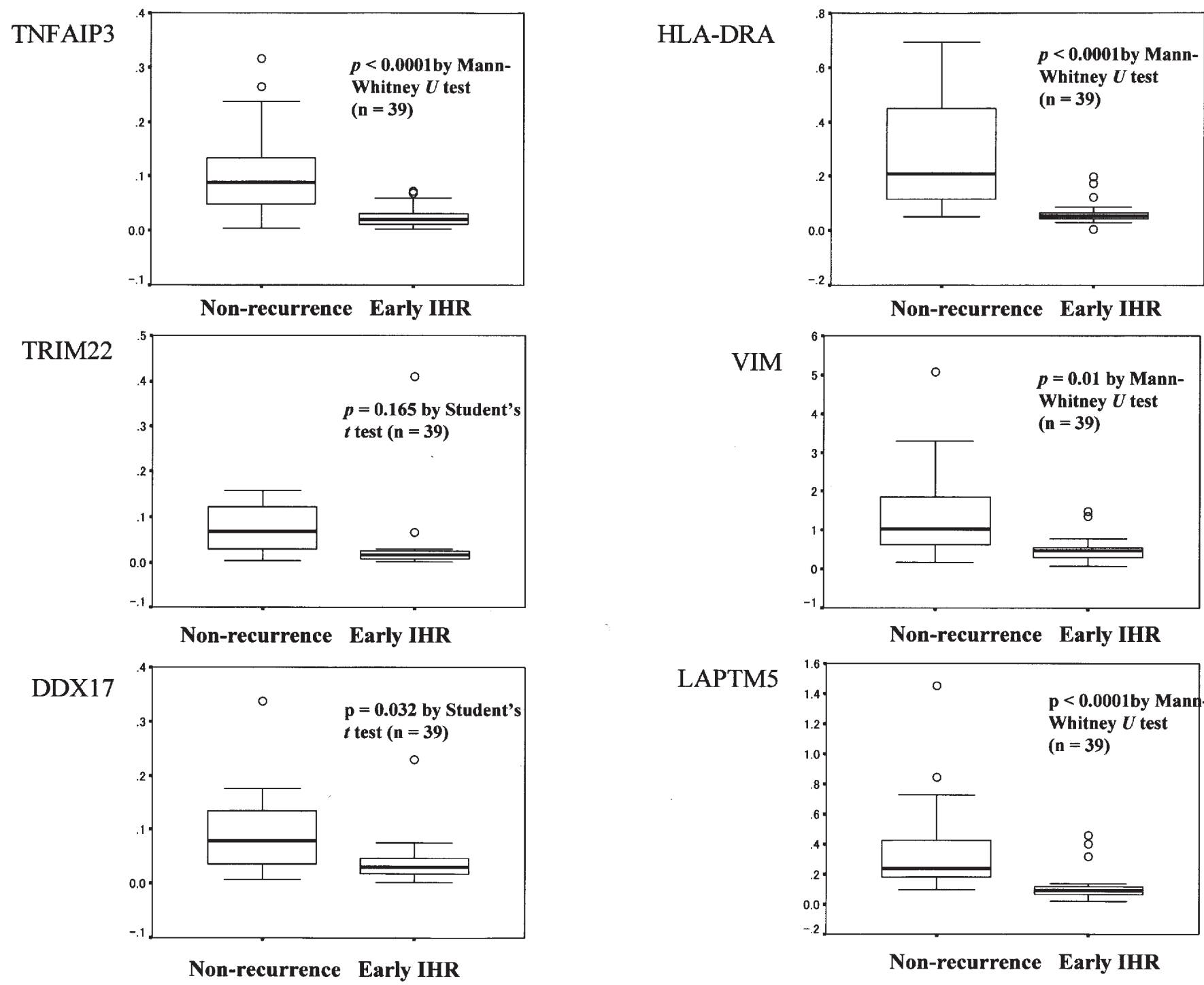

VIM

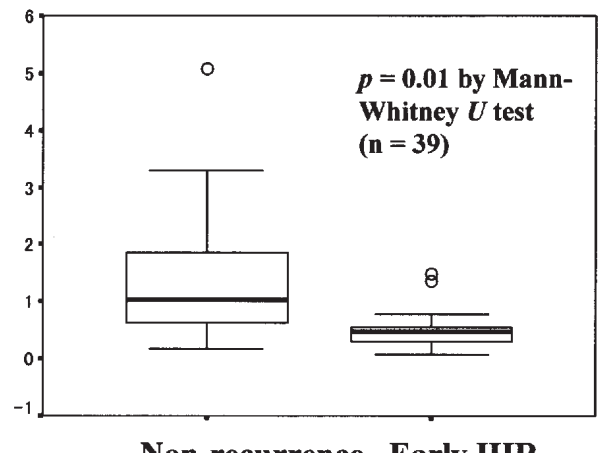

LAPTM5

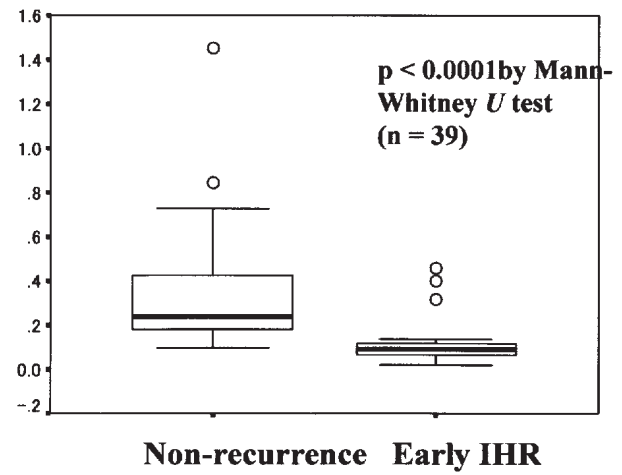

Figure 3. Box and whisker plot of expression patterns of the 6 genes in HCCs with early IHR and those without recurrence. Gene symbols were obtained from Entrez Gene (http://www.ncbi.nlm.nih.gov/entrez/query.fcgi?db=gene).

The specificity of the products was documented with analysis of the melting temperature (data not shown). The primers used in this study were GAPDH-S, 5'-TGGTATCGTGGAA GGACTCA-3' and GAPDH-AS, 5'-GGGGCCATCCACAGT CTT-3'; TNFAIP3-S, 5'-TGCACACTGTGTTTCATCGAG-3' and TNFAIP3-AS, 5'-ACGCTGTGGGACTGACTTTC-3'; HLADRA-S, 5'-CAAGGGATTGCGCAAAAG-3' and HLADRA-AS, 5'-GATCTTCTCTCTAAGAAACACCATCA3'; TRIM22-S, 5'-CTAAAGAGTGTATTCCGAGTACCAG AT-3' and TRIM22-AS, 5'-GCATCACGTCCACCCAGTA-3'; DDX17-S, 5'-AAACTGATGCAGCTTGTGGA-3' and DDX17-AS, 5'-CGGTAACGAGAACGACCAC-3'; VIM-S, 5'-TACAGGAAGCTGCTGGAAGG-3' and VIM-AS, 5'-TC AGGGAGGAAAAGTTTGGA-3'; and LAPTM5-S, 5'-TCA TCTTTTCCATCGCCTTC-3' and LAPTM5-AS, 5'-TTCTC CTCCACCGAGTTCAT-3'. The predicted product sizes were 70, 76, 87, 94, 71, 69, and 109 bp for GAPDH, TNFAIP3, HLADRA, TRIM22, DDX17, VIM and LAPTM5, respectively. Construction of predictor with training set. In the leave-oneout method, we selected 38 HCC samples from the 39 training HCC samples and constructed the Fisher linear classifier (FLC)
(6) of $\mathrm{N}$ genes with the 38 samples. The performance of the constructed classifier on the remaining one HCC sample was tested, and this procedure was repeated 39 times. Each exhaustive search was performed for all combinations $(n=2$, $3,4,5$ and 6 ) of the 6 genes. As a result, we determined that the gene combination of HLA-DRA, DDX17 and LAPTM5 minimized the leave-one-out error rate and maximized prediction (data not shown). The formula for the optimal predictor given by FLC was follows: $\mathrm{F}(\mathrm{x})=9.783538 \times H L A-D R A+$ 8.288929 x $D D X 17$ - 1.684217 x LAPTM5 - 1.547365. If $\mathrm{F}(\mathrm{x})<0$, our predictor predicted early IHR. If $\mathrm{F}(\mathrm{x})>0$, it predicted non-recurrence.

Evaluation of the performance of the optimal predictor on an independent test set. Finally, the performance of our final predictive system $\mathrm{F}(\mathrm{x})$ was tested on 43 independent $\mathrm{HCC}$ samples (test set) (Fig. 1).

Statistical analysis. The reproducibility of the expression patterns of the 12 genes between the 2 distinct array data sets was confirmed with Pearson's correlation coefficient. 
Table II. Independent risk factors for early intrahepatic recurrence.

\begin{tabular}{lcccc}
\hline Variable & Regression coefficient & Standard error & Risk ratio (95\% CI) & P-value \\
\hline $\mathrm{F}(\mathrm{x})<0$ & 2.61 & 0.95 & $13.6(2.1-88.1)$ & $\mathrm{P}=0.006$ \\
Stage IIIA & 1.37 & 0.59 & $3.9(1.2-12.5)$ & $\mathrm{P}=0.019$ \\
\hline
\end{tabular}

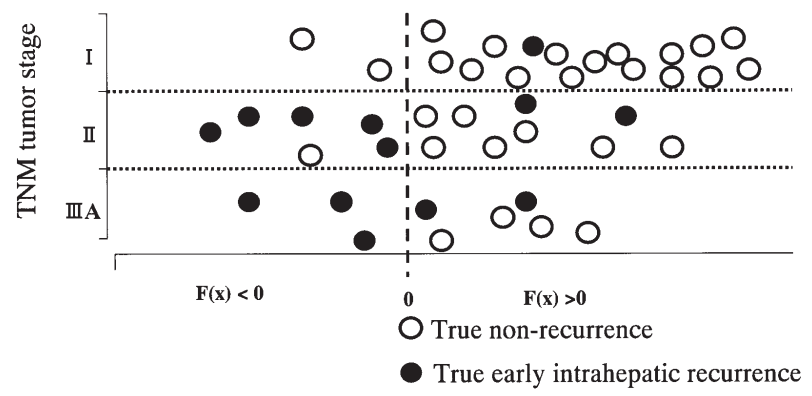

Figure 4. Performance of the 3-gene predictor on 43 independent test samples. $\mathrm{F}(\mathrm{x})$, formula computed with the Fisher linear classifier. If $\mathrm{F}(\mathrm{x})<0$, our 3-gene predictor predicted early IHR. If $\mathrm{F}(\mathrm{x})>0$, it predicted non-recurrence. In total, our predictor correctly predicted early IHR or non-recurrence in $35(81.4 \%)$ of $43 \mathrm{HCCs}$ in the independent test set. The predictive accuracy was $84.2 \%$ (16/19) for stage-I HCCs, 80.0\% (12/15) for stage-II HCCs, and 77.8\% (7/9) for stage-IIIA HCCs.

QRT-PCR data for the 6 genes were analyzed by Student's t-test and Mann-Whitney U test. We carried out multivariate analysis to assess independent factors for early IHR in the 43 HCC samples using the logistic regression model (SPSS 11.0J software, SPSS, Inc., Chicago, IL). Five variables $[\mathrm{F}(\mathrm{x})$, tumor size, venous invasion, number of primary lesions and TNM stage] were entered into a backward elimination model. Each model was tested for goodness-of-fit by $-2 \log$ likelihood and chi-square at each step. $\mathrm{P}<0.05$ was accepted as statistically significant.

\section{Results}

During the follow-up periods, 17 patients with HCC (43.6\%) and 13 patients with $\mathrm{HCC}(30.2 \%)$ in the training and test sets, respectively, had early IHR within 1 year after curative hepatectomy (Table I). We found that tumor size, venous invasion and TNM stage were associated with early IHR and that the number of primary lesions tended to be associated with early IHR in the HCC training set (Table I). In the HCC test set, TNM stage and the number of primary lesions were associated with early IHR, but tumor size was not associated with early IHR (Table I). Thus, there was little difference in patient characteristics between the HCC training and test sets.

To validate the performance of our QRT-PCR analysis, we compared expression of the 6 genes between HCCs with early IHR and those without early IHR in the training set. The levels of TNFAIP3, HLADRA, DDX17, VIM and LAPTM5 were significantly lower in HCC with early IHR than in HCC without (Fig. 3). Although there was no statistical difference in levels of TRIM22 between the 2 groups, the average levels were 1.85-fold lower in HCC with early IHR than in HCC without (Fig. 3). Thus, our QRT-PCR analysis reproduced the performance of the 6 genes from previous microarray analysis (6).

By screening for all combinations $(n=2-6)$ of the 6 genes, we identified an optimal combination of 3 genes (HLADRA, $D D X 17$ and LAPTM5) that minimized the leave-one-out error for prediction of early IHR in the training set. The 3-gene predictor $[\mathrm{F}(\mathrm{x})]$ constructed with the Fisher linear classifier correctly predicted early IHR or non-recurrence in 35 (81.4\%) of $43 \mathrm{HCCs}$ in the independent test set (Fig. 4). It had a positive predictive value (PPV) of $72.7 \%$, negative predictive value (NPV) of $84.4 \%$, sensitivity of $61.5 \%$ and specificity of $90.0 \%$. The predictive accuracy was $16(84.2 \%)$ of 19 stage-I HCCs, $12(80.0 \%)$ of 15 stage-II HCCs and $7(77.8 \%)$ of 9 stage-IIIA HCCs, respectively (Fig. 4). Thus, our predictor worked well for early-stage HCC. There was no recurrence in $18(94.7 \%)$ of 19 stage-I HCCs or in $12(50.0 \%)$ of 24 HCCs of stages IIIIIA. The TNM staging system had a poor PPV of $49.9 \%$, whereas it had a good NPV of $94.7 \%$. When 5 variables $[\mathrm{F}(\mathrm{x})$, tumor size, venous invasion, number of primary lesion and TNM stage] were entered into a backward elimination model of logistic regression, the 3-gene predictor was an independent risk factor for early IHR in the HCC test set [risk ratio, 13.6; $95 \%$ confidence interval $(\mathrm{CI}), 2.1-88.1 ; \mathrm{p}=0.006]$ (Table II).

\section{Discussion}

DNA microarray technology has created a new means by which patients at distinct risk for recurrence of HCC can be identified (18). Although microarray analysis is a powerful technique, it is still relatively laborious and expensive and requires careful and complicated data analysis. In particular, most DNA microarray-based predictors have required large numbers of gene signatures. As a result, arrays remain a high-dimensional and expensive strategy. To confirm microarray results in larger cohorts (10), a cheaper, easier, and faster method, such as QRT-PCR, is needed. We previously developed an accurate predictor of IHR using only 12 genes (6). To extend this result, in our present study, we developed a 3-gene PCR-based predictor that will cost less than 10 US dollars per sample, and that accurately predicted early IHR in a set of independent HCC samples.

PCR technology has allowed for quantitative analysis of target genes in small amounts of DNA or RNA. Some investigators have used this technology to detect cancerspecific transcripts originating from HCC cells circulating in the bloodstream or HCC tissues (19-21). Cheung et al (22) used RT-PCR to select the Claudin-10 gene from a 23,000- 
gene DNA microarray and showed that this gene has high predictive value for outcome of HCC patients after surgery. These studies (20-23) showed that the level of one specific gene correlated with the prognosis of HCC or detected transcripts such as $\alpha$-fetoprotein and telomerase reverse transcriptase in blood from HCC patients; however, the predictive powers of these genes for recurrence was not satisfactory for clinical use. There has been only one study (7) of a PCR-based predictor that accurately classified early IHR or non-recurrence in independent HCC samples in a training-validation manner. The 20-gene PCR-based predictor of Kurokawa et al (7) correctly predicted early IHR or non-recurrence in 29 (72.5\%) of $40 \mathrm{HCCs}$. In the present study, there were some differences in patient characteristics between the HCC training and test sets; however, the predictive accuracy of our 3-gene predictor was still $81.4 \%$ (35/43). This degree of accuracy was superior to that of the 20-gene predictor by Kurokawa et al (7) and, to our knowledge, it is the most robust PCR-based predictor for early IHR of HCC after hepatectomy. This superiority might be due to our unique gene selection procedure in which the combination of genes was considered and 2 distinct DNA microarray platforms were used to select the most reliable genes. Given that PCR technology can analyze target genes in small amounts of DNA or RNA, our methodology may enable us to evaluate the metastatic potential of HCC preoperatively from small amounts of the biopsy specimens. The 3 genes used in our predictive system had no overlap with signature genes linked to patient backgrounds, such as hepatitis virus type, tumor differentiation grade or coexisting liver diseases (i.e., cirrhosis, hepatitis or normal liver) in our series $(11,12,14)$. In addition, our 3 -gene predictor was an independent risk factor for early IHR in the HCC test set. Thus, the lack of relation of the 3 genes to patient characteristics accounts for the high predictive value.

There are likely 2 opposite strategies for developing a multigene predictor for cancer outcome. One strategy decreases the dimensionality (i.e., the number of genes) in the multigene predictor, and the other strategy uses as many genes as possible. Our current study aimed to develop a predictor of early IHR of HCC with the decreased dimensionality strategy. It was reported that among the 36 feature genes obtained from data of several DNA microarray studies, measurement of the expression of only 6 genes by QRT-PCR was sufficient to predict overall survival for diffuse large-B-cell lymphoma (23). Likewise, Endoh et al (24) screened for genes, published in DNA microarray data and developed an 8-gene predictive model for outcome of pulmonary adenocarcinoma patients by QRT-PCR that worked well in an independent set of pulmonary adenocarcinomas. Ma et al (25) identified, from 22,000 genes, 2 genes related to outcome of breast cancer patients after tamoxifen treatment, and the 2-gene expression ratio yielded by QRT-PCR accurately predicted the treatment outcome of breast cancer patients. In conjunction with our present finding, these reports support the efficacy of a lowdimensional predictor for IHR of HCC in clinical practice.

As mentioned above, opposite conclusions were also drawn from several studies. For example, the 2-gene predictive model (25) for breast cancer patients could not be replicated in a new cohort of patients by other investigators (26). RTPCR analysis by Shultz et al (27) also showed a lack of reproducibility of a DNA microarray study for association with recurrence of urothelial cell carcinoma. It is apparent that patient heterogeneity may be one explanation for the lack of overlap between predictive signatures from different studies or institutes. To circumvent heterogeneity, Roepman et al (28) suggested that it is necessary to integrate information for many genes into the predictor. This concept might lead to development of a multi-gene predictor that can be used broadly; however, they did not evaluate the predictive performance in independent samples, and the predictive power may be decreased due to inclusion of many ineffective genes. Our current approach successfully decreased the number of genes while retaining predictive performance. As a result, our 3gene predictor yielded a high accuracy on an independent cohort. Such a robust predictor with a small set of genes is advantageous for the clinical setting. Further large-scale studies are needed to elucidate the degree to which our 3-gene predictor works in predicting early IHR.

\section{Acknowledgements}

Grant sponsors were the Ministry of Education, Culture, Sports, Science and Technology (no. 18390366, no. 17591406 and Knowledge Cluster Initiative); the Venture Business Laboratory of Yamaguchi University; the New Energy and Industrial Technology Development Organization (no. 03A02018a).

\section{References}

1. Parkin DM, Bray FI and Devesa S: Cancer burden in the year 2000. The global picture. Eur J Cancer 37 (suppl 8): S4-S66, 2001.

2. El-Serag HB and Mason AC: Rising incidence of hepatocellular carcinoma in the United States. N Engl J Med 340: 745-750, 1999.

3. Llovet JM, Burroughs A and Bruix J: Hepatocellular carcinoma. Lancet 362: 1907-1917, 2003.

4. Poon RT, Fan ST, Ng IO, Lo CM, Liu CL and Wong J: Different risk factors and prognosis for early and late intrahepatic recurrence after resection of hepatocellular carcinoma. Cancer 89: 500-507, 2000.

5. Yamamoto J, Kosuge T, Takayama T, Shimada K, Yamasaki S, Ozaki H, Yamaguchi N and Makuuchi M: Recurrence of hepatocellular carcinoma after surgery. Br J Surg 83: 1219-1222, 1996.

6. Iizuka N, Oka M, Yamada-Okabe H, et al: Oligonucleotide microarray for prediction of early intrahepatic recurrence of hepatocellular carcinoma after curative resection. Lancet 361: 923-929, 2003.

7. Kurokawa Y, Matoba R, Takemasa I, et al: Molecular-based prediction of early recurrence in hepatocellular carcinoma. J Hepatol 41: 284-291, 2004.

8. Schena M, Shalon D, Davis RW and Brown PO: Quantitative monitoring of gene expression patterns with a complementary DNA microarray. Science 270: 467-470, 1995.

9. Lee JS, Chu IS, Heo J, et al: Classification and prediction of survival in hepatocellular carcinoma by gene expression profiling. Hepatology 40: 667-676, 2004

10. Ntzani EE and Ioannidis JP: Predictive ability of DNA microarrays for cancer outcomes and correlates: an empirical assessment. Lancet 362: 1439-1444, 2003.

11. Iizuka N, Oka M, Yamada-Okabe H, et al: Comparison of gene expression profiles between hepatitis B virus- and hepatitis C virus-infected hepatocellular carcinoma by oligonucleotide microarray data on the basis of a supervised learning method. Cancer Res 62: 3939-3944, 2002.

12. Iizuka N, Oka M, Yamada-Okabe H, et al: Differential gene expression in distinct virologic types of hepatocellular carcinoma: association with liver cirrhosis. Oncogene 22: 3007-3014, 2003. 
13. Matoba K, Iizuka N, Gondo T, et al: Tumor HLA-DR expression linked to early intrahepatic recurrence of hepatocellular carcinoma. Int J Cancer 115: 231-240, 2005.

14. Iizuka N, Oka M, Yamada-Okabe H, et al: Self-organizingmap-based molecular signature representing the development of hepatocellular carcinoma. FEBS Lett 579: 1089-1100, 2005.

15. Iizuka N, Tsunedomi R, Tamesa T, et al: Involvement of c-mycregulated genes in hepatocellular carcinoma related to genotypeC hepatitis B virus. J Cancer Res Clin Oncol 132: 473-481, 2006.

16. Tsunedomi R, Iizuka N, Yamada-Okabe H, et al: Identification of ID2 associated with invasion of hepatitis $\mathrm{C}$ virus-related hepatocellular carcinoma by gene expression profile. Int J Oncol 29: 1445-1451, 2006.

17. Neuvians TP, Gashaw I, Sauer CG, et al: Standardization strategy for quantitative PCR in human seminoma and normal testis. J Biotechnol 117: 163-171, 2005.

18. Lee JS and Thorgeirsson SS: Genome-scale profiling of gene expression in hepatocellular carcinoma: classification, survival prediction, and identification of therapeutic targets. Gastroenterology 127: S51-S55, 2004.

19. Ijichi M, Takayama T, Matsumura M, Shiratori Y, Omata M, and Makuuchi M: alpha-Fetoprotein mRNA in the circulation as a predictor of postsurgical recurrence of hepatocellular carcinoma: a prospective study. Hepatology 35: 853-860, 2002.

20. Witzigmann H, Geissler F, Benedix F, Thiery J, Uhlmann D, Tannapfel A, Wittekind C and Hauss J: Prospective evaluation of circulating hepatocytes by alpha-fetoprotein messenger RNA in patients with hepatocellular carcinoma. Surgery 131: 34-43, 2002.
21. Waguri N, Suda T, Nomoto M, et al: Sensitive and specific detection of circulating cancer cells in patients with hepatocellular carcinoma; detection of human telomerase reverse transcriptase messenger RNA after immunomagnetic separation. Clin Cancer Res 9: 3004-3011, 2003.

22. Cheung ST, Leung KL, Ip YC, et al: Claudin-10 expression level is associated with recurrence of primary hepatocellular carcinoma. Clin Cancer Res 11: 551-556, 2005.

23. Lossos IS, Czerwinski DK, Alizadeh AA, Wechser MA, Tibshirani R, Botstein D and Levy R: Prediction of survival in diffuse large-B-cell lymphoma based on the expression of six genes. N Engl J Med 350: 1828-1837, 2004.

24. Endoh H, Tomida S, Yatabe Y, et al: Prognostic model of pulmonary adenocarcinoma by expression profiling of eight genes as determined by quantitative real-time reverse transcriptase polymerase chain reaction. J Clin Oncol 22: 811-819, 2004.

25. Ma XJ, Wang Z, Ryan PD, et al: A two-gene expression ratio predicts clinical outcome in breast cancer patients treated with tamoxifen. Cancer Cell 5: 607-616, 2004.

26. Reid JF, Lusa L, De Cecco L, et al: Limits of predictive models using microarray data for breast cancer clinical treatment outcome. J Natl Cancer Inst 97: 927-930, 2005.

27. Schultz IJ, Wester K, Straatman H, et al: Prediction of recurrence in Ta urothelial cell carcinoma by real-time quantitative PCR analysis: a microarray validation study. Int J Cancer 119: 1915-1919, 2006.

28. Roepman P, Kemmeren P, Wessels LF, Slootweg PJ and Holstege FC: Multiple robust signatures for detecting lymph node metastasis in head and neck cancer. Cancer Res 66: 2361-2366, 2006. 